\title{
Feeling multiple edges: the tactile perception of short ultrasonic square reductions of the finger-surface friction*
}

\author{
D. Gueorguiev ${ }^{1}$, E. Vezzoli ${ }^{2}$, T. Sednaoui ${ }^{2}$, L. Grisoni ${ }^{1}$, B. Lemaire-Semail ${ }^{2}$, Member, IEEE
}

\begin{abstract}
This study investigates human perception of tactile feedback using ultrasonic lubrication, in situation where feedback is provided using short frictional cues of varying duration and sharpness. We asked participants to discriminate the transition time and duration of short square ultrasonic reductions of friction. They proved very sensitive to discriminate millisecond differences in this two parameters with the average psychophysical thresholds being $2.4 \mathrm{~ms}$ for discriminating duration and $2.06 \mathrm{~ms}$ for transition time. A second experiment focused on participant's perception of square friction reductions of variable transition time and duration and we found that for durations of the stimulation larger than $90 \mathrm{~ms}$, participants often perceived 3 or 4 edges when only two stimulations were presented while they consistently felt 2 edges for signals shorter than $50 \mathrm{~ms}$. These results confirm the sensitivity of touch to transient frictional cues on smooth surfaces and raises the question of how such cues are processed by the neural mechanisms mediating the perception of friction. Moreover, the knowledge of how potentially ambiguous frictional cues are resolved is central to the implementation tactile patterns on friction-based displays with haptic feedback as well as to the definition of unambiguous core frictional blocks.
\end{abstract}

\section{INTRODUCTION}

The last few years have seen the emergence of mobile devices and tactile interfaces with friction based haptic feedback. The development of these novel interfaces raised the interest in touch based human-machine interactions and highlighted the lack of natural touch feedback in the existing generation of tactile displays. As the applications for controlled friction modulation are ever-growing [1]-[3], the development of a high-fidelity strategy for tactile rendering is needed but faces the limitation that little is known about the sensory mechanisms mediating our perception of frictional cues. Currently, multiple solutions are being explored to deliver improved haptic feedback on existing mobile platforms such as smartphones or tablets. One such feedback technology, vibrotactile stimulation, is already incorporated on most platforms but only provides a general vibration sensation to the hand and finger of users. To improve upon this, tactile based solutions have been proposed in recent years such as electrovibration [4] and ultrasonic lubrication [5]-[7]. These technologies have very different means of action but ultimately affect the dynamic friction between the finger and the tactile plate in a similar manner [8]. To implement realistic shapes and textures, it is also essential to understand which components of the frictional signal are critical for tactile

\footnotetext{
*This study was funded by the FP7 Marie Curie Initial Training Network PROTOTOUCH, grant agreement No. 317100

D. Gueorguiev (e-mail: david.gueorguiev@inria.fr) and L. Grisoni (email: laurent.grisoni@inria.fr) are with INRIA Lille-Nord Europe, MINT team, F-59650 Villeneuve d'Asq, France
}

sensation and how to scale their intensities according to the dynamics of the interaction. It is well-known that the fingersurface friction has an essential role in tactile perception [6] and that the perception of edges depends on the shear strains induced by the resulting compression of the skin [9]. While much research has focused on periodic signals [10], [11], little is known about how virtual geometric features (e.g: buttons, edges, patterns) should be designed on friction-based tactile displays. Recent research has shown that touch is very at accurate at perceiving the changes in friction even when they are evanescent [12], which enables the use of transient signals in order to generate meaningful feedback [1]. A better understanding of the tactile perception of short reductions of friction will also contribute to the definition of a tactile pixel, which is currently a major challenge for the development of tactile displays with haptic feedback [13], [20]. In our study, we use an ultrasonic device with high temporal resolution and controlled amplitude of ultrasonic vibration [14] to investigate the human perception of short square reductions of friction. In a first experiment, we quantitatively investigate two parameters related to the perception of square waves: the transition time, which is the time needed to reach the desired amplitude of ultrasonic vibration and recover from it, and the duration of the uniform reduction of friction. In a second experiment, we asked the participants to count the number of edges they felt while exploring two consecutive stimulations, which displayed a wide range of durations and transition times.

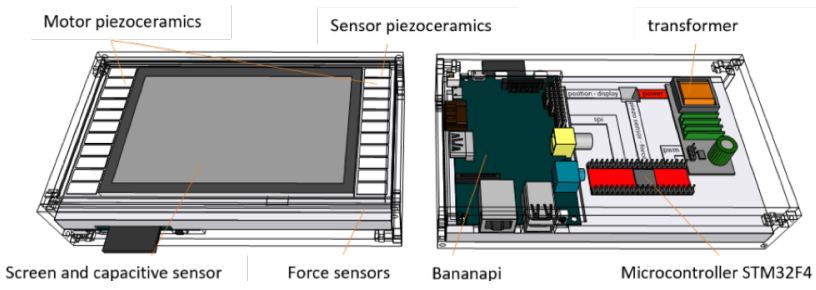

Figure 1. Illustration of the ultrasonic device E-ViTa, which was used to deliver de ultrasonic square stimulations in the two experiments.

\section{MATERIALS AND METHODS}

\section{A. Participants}

Data were collected from 12 healthy volunteers aged between 25 and 40 ( 2 females) in experiment 1. Participants were wearing active noise-cancelling headphones (Quiet Comfort 25, Bose, USA) in order to prevent potential interference from auditory cues. Six other healthy volunteers

B. Lemaire-Semail, E. Vezzoli and T. Sednaoui are with Univ. Lille, Centrale Lille, Arts et Metiers ParisTech, HEI, EA 2697 - L2EP - Laboratoire d'Electrotechnique et d'Electronique de Puissance, F-59000 Lille, France. (betty.semail@polytech-lille.fr) 
aged between 23 and 32 ( 1 female) participated in experiment 2. All participants gave written informed consent. The investigation conformed to the principles of the Declaration of Helsinki and experiments were performed in accordance with relevant guidelines and regulations.

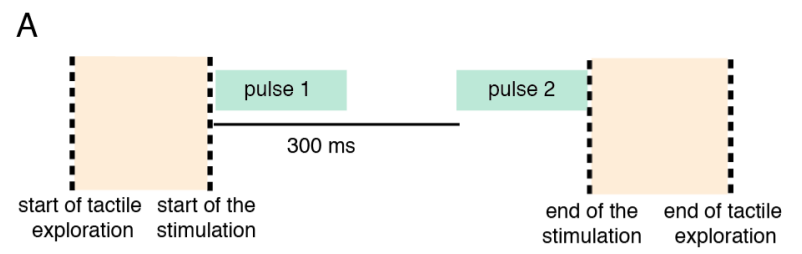

B

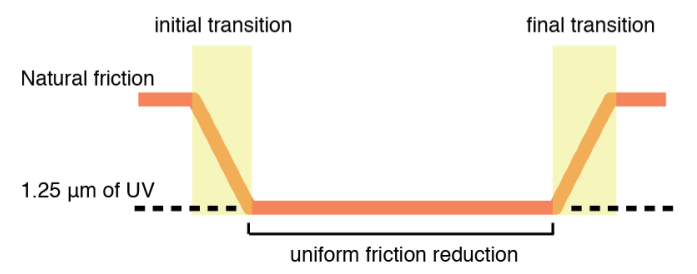

Figure 2. A/ In all trials of both experiments, two consecutive identical square reductions were delivered when the finger reached a predefined location on the screen B/ Each square reduction implemented by ultrasonic vibration (UV) depends on two parameters: its transition time, which relates to its sharpness and the duration of the central interval of uniform friction reduction.

\section{B. Experimental set-up}

In order to control the modulation frequency and the vibration amplitude of the haptic plate, a high-resolution ultrasonic device was used (Fig. 1). This system includes both visual and haptic feedback. Finger touch position is directly acquired using a capacitive touch screen. Computation and control of the experiment is separated in two parts: a "High level" signal using the banana pi (Shenzhen LeMaker Technology Co. Ltd, China) single board computer featuring a $1 \mathrm{GHz}$ ARM Cortex-A7 dual-core CPU with $1 \mathrm{~GB}$ of ram. A "Low Level" signal generation is implemented in a separate DSP microcontroller (stm32f4, STMicroelectronics, France) running at $164 \mathrm{MHz}$. In this setup "High level" computing refers to the display of the instruction to the user, selection of the haptic signal commands and storage of the results. The signal generation microcontroller for its part applies commands from the board computer to create the necessary waveforms for the friction modulation. The communication between the microcontroller and the single board $\mathrm{pc}$ is provided by an SPI bus working at $10 \mathrm{kHz}$. In order to ensure the fastest amplitude transition time in this study, an external amplifier is used to drive the piezoceramic motors as in [15].

The single board computer is connected to a 5 inches flat capacitive touch screen (Banana-LCD-5"-TS, Marel, China) providing the finger position input and display output, where the sampling frequency of the finger position is $62 \mathrm{~Hz}$. This LCD display gives visual confirmation of the experiment goals during the measures. A second visual system using a computer screen is used to display comfortably the controls of the experiments to each participant. The ultrasonic vibrating plate implemented in the device is specifically designed to provide the best modulation bandwidth. The glass plate measures $154 \times 81 \times 1.6 \mathrm{~mm}$ and resonates at $60750 \mathrm{~Hz}$, where the half length of the vibration mode is $8 \mathrm{~mm}$. 22 piezoceramics, $14 \times 6 \times 0.5 \mathrm{~mm}$, are mounted at the side of the plate along the extremum of deformation, 20 used as motors and 2 as vibration sensors.

\section{General procedure}

Two experiment were performed, in which each tactile exploration consisted in two identical consecutive square friction reductions delivered with $300 \mathrm{~ms}$ interval, (Fig 2A). The stabilized ultrasonic amplitude was set at $1.25 \mu \mathrm{m}$ (Fig. 2B). The choice of two consecutive presentations of an identical signal was made to enable a better comparison of the signals in experiment 1 as well as to provide more options for the counting task performed in experiment 2 .

Experiment 1 consisted in a two alternatives forced-choice task (2AFC) in which the participants had to compare varying ultrasonic square signals to a reference signal either in terms of sharpness or duration. In the sharpness task, participant had to pick the sharper out of two proposed stimulations, which they could explore up to three times before answering. One of the two stimuli, randomly presented first or second had always a $0.3 \mathrm{~ms}$ transition time and $10 \mathrm{~ms}$ duration while the comparison stimulus was of the same duration but with different transition times: $0.6,1,1.8,2.7$, or $4.2 \mathrm{~ms}$. The same experimental protocol was implemented to discriminate between durations. A reference signal of $0.8 \mathrm{~ms}$ duration and $0.5 \mathrm{~ms}$ transition time was to be compared with signals having the same transition time but different durations: 1.6, 2.4, 3.2, 4 or $4.8 \mathrm{~ms}$. In both experiments, each pair of stimuli was presented 10 times in a pseudo-random order for a total number of 50 trials. The psychophysical thresholds computed in experiment 1 were evaluated by fitting a logistic psychometric function based on the method of maximum likelihood to the psychophysical performance of the participants. The fitting was implemented by using the version 1.81 of the Palamedes toolbox [16].

In experiment 2, participants were asked to count the number of edges that they felt during a tactile exploration of an ultrasonic signal. The ultrasonic signal was always composed by two square reductions of friction that were played at an interval of $300 \mathrm{~ms}$ but had different durations (30, $50,70,90,110 \mathrm{~ms})$ and transition times $(0.5,1.2$ or $2.0 \mathrm{~ms})$. Each combination of duration and transition time was randomly presented 10 times to the participants (150 trials in total). At each trial, participants explored the signal only once and were forced to respond by typing a number between 0 and 6 in order to report the number of edges that they felt.

TABLE I. NUMBER OF PERCEIVED EDGES

\begin{tabular}{|c|c|c|c|}
\hline \multirow{2}{*}{$\begin{array}{c}\text { Duration of } \\
\text { the signal }\end{array}$} & \multicolumn{3}{|c|}{$\begin{array}{c}\text { Average number of perceived edges depending on } \\
\text { transition time }\end{array}$} \\
\cline { 2 - 4 } & $\mathbf{0 . 5} \boldsymbol{m s}$ & $\mathbf{1 . 2} \boldsymbol{m s}$ & $\mathbf{2 . 0} \boldsymbol{~} \boldsymbol{s}$ \\
\hline $30 \mathrm{~ms}$ & $2.03 \pm 0.21$ & $2.15 \pm 0.10$ & $2.08 \pm 0.22$ \\
\hline $110 \mathrm{~ms}$ & $2.70 \pm 0.28$ & $3.13 \pm 0.38$ & $2.88 \pm 0.50$ \\
\hline
\end{tabular}


A

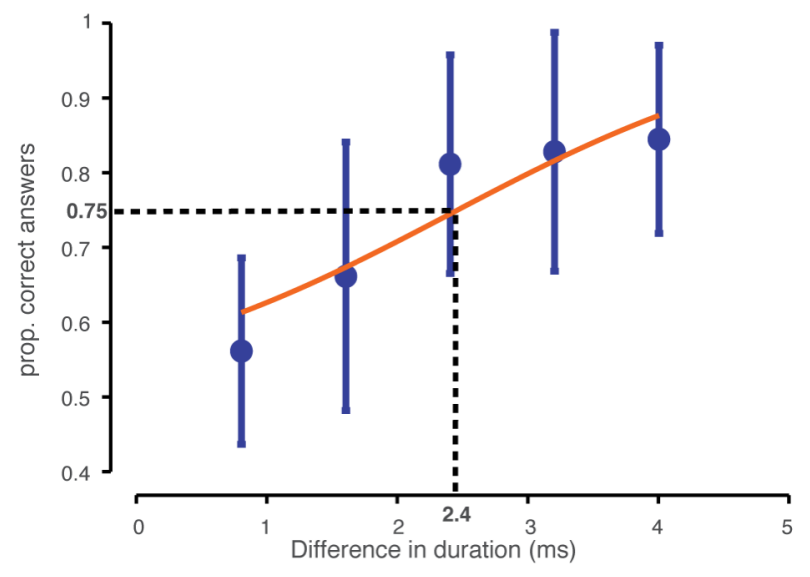

B

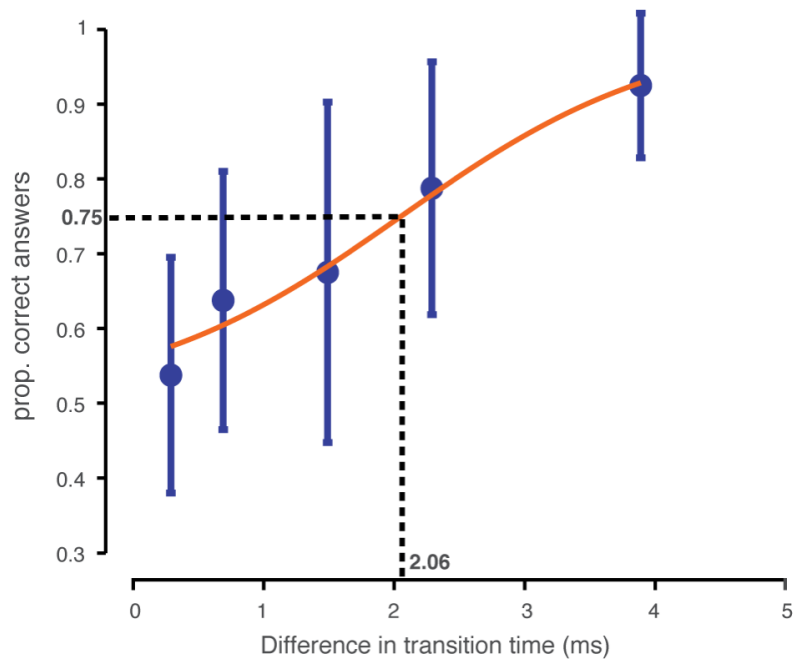

Figure 3. A/ Just noticeable difference of the duration of the square friction reduction. The blue error bars represent the standard deviation across participants and the red line is a fit of a logistic psychometric function to the participants' averaged answers. B/ Just noticeable difference for the transition time of a square friction reduction. The blue error bars represent the standard deviation and the red line is a fit of a logistic psychometric function to the participant's averaged answers.

\section{RESULTS}

The participants' psychophysical threshold for detecting differences between stimuli is commonly defined as the difference between the reference and a comparison stimulus that is correctly perceived in $75 \%$ of the trials. This threshold is also termed $75 \%$ just noticeable difference (JND). Thus, the average proportion of correct answers was computed for each pair of stimuli and the $75 \%$ JND was estimated by fitting a logistic psychometric function to the averaged answers across participants and interpolating the difference in transition time or duration at the threshold value. Participants showed a very good capacity to distinguish between signals with differing duration and transition time. When comparing signals of different durations against the reference stimulus of $0.8 \mathrm{~ms}$ duration and $0.5 \mathrm{~ms}$ transition time, the $75 \%$ JND was found to be $2.4 \mathrm{~ms}$ (Fig 3A). When comparing signals of different transition times against the reference stimulus of $10 \mathrm{~ms}$ duration and $0.3 \mathrm{~ms}$ transition time, the $75 \%$ JND was estimated at $2.06 \mathrm{~ms}$ (Fig 3B). These results show that human users are capable of sensing very small differences in the duration and the sharpness of an ultrasonic reduction of friction.

The high sensitivity to millisecond differences between frictional signals suggested that it might be possible that a prolonged square wave reduction is no longer perceived as a unique stimulation but that the initial (falling friction) and final transition (recovery to the natural friction) would be felt separately. Thus, we investigated if a square reduction of friction could be felt as two distinct stimulations by asking the participants to count the number of edges that they felt during stimulations with durations from 30 to $110 \mathrm{~ms}$ and transition times from 0.5 to $2.0 \mathrm{~ms}$ (Fig. 4) consecutively presented two times. Results showed indeed that for a constant number of delivered square reductions of friction (2), the number of distinct edges felt by participants was increasing for larger durations. A statistically significant increase of the number of felt edges between $30 \mathrm{~ms}$ and $110 \mathrm{~ms}$ of duration was observed for each of the tested transition times (paired t-test with $\mathrm{p}<0.5$ for the three tested transition times and $p=0.009$ when the three transition times are merged). At $30 \mathrm{~ms}$, participants felt two edges most of the time and no significant deviation was observed ( $t$-test against two with $p=0.73 p=0.17$ and $p=0.43$ ) while at $110 \mathrm{~ms}$, the answers were significantly larger than two (t-test against two with respectively $\mathrm{p}=0.002, \mathrm{p}=0.001$ and $\mathrm{p}=0.011)($ see also table 1$)$.

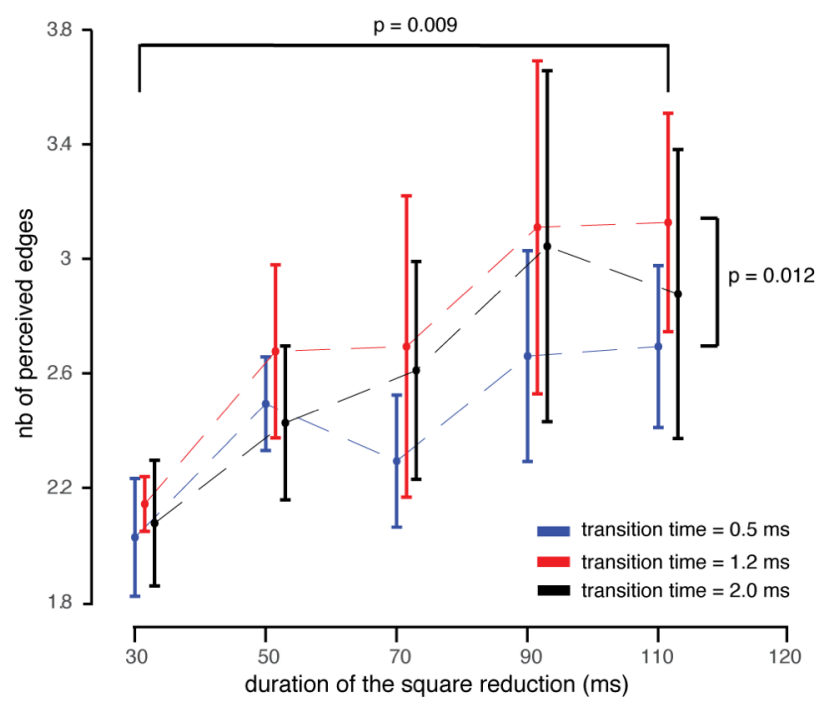

Figure 4. The average number of perceived edges per trial plotted against the duration of the signal for each of the three tested transition times $(0.5$ $\mathrm{ms}, 1.2 \mathrm{~ms}$ and $2 \mathrm{~ms}$ ). The error bars represent the standard deviation across participants.

We found that sharpness also influenced significantly the increase of the number of perceived edges for larger transition times since the condition with $0.5 \mathrm{~ms}$ of transition time showed a less pronounced increase of the number of perceived edges than the other two conditions (one-way ANOVA: $p=0.012$ ). This means that when the "borders" of the ultrasonic square signal are sharper, the probability of perceiving two edges instead of one for a square signal is reduced. Moreover, it is 
interesting to notice that for larger durations of the square reduction, the number of reported stimulations is not predominantly 4 but is uniformly distributed between 2, 3 and 4 and participants often reported having felt 3 edges (Fig. 5). These results suggest that the doubling of the perception is sometimes only felt for one of the two square reductions that are presented to the participant. It would be interesting to further investigate if the doubling of the perceived edges is dependent on the presentation order (first or second) of the ultrasonic square reduction of the friction.
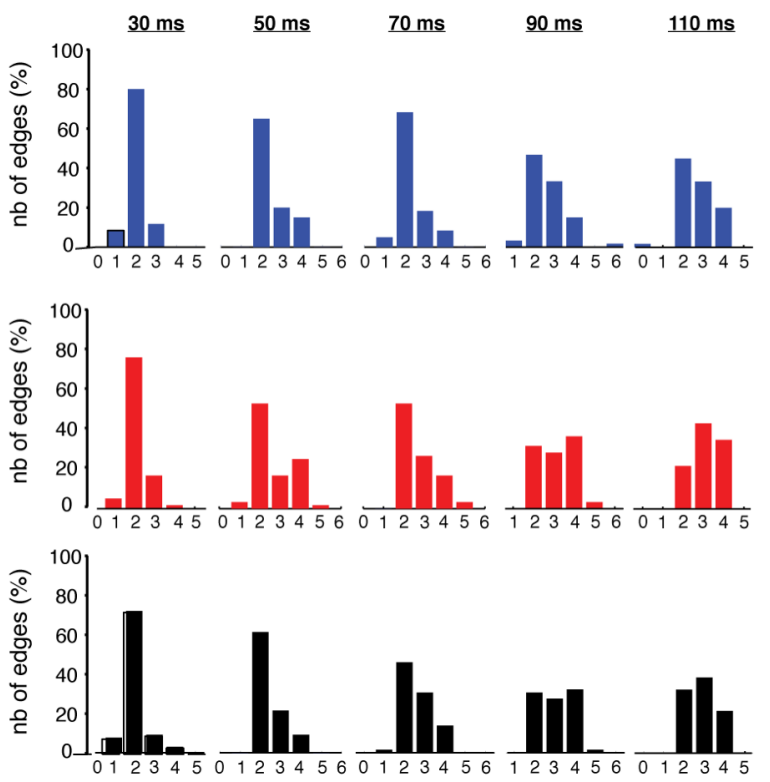

Figure 5. The average percentage of each possible answer (from 0 to 6) across participants plotted for all the possible durations and the three tested transition times. The perceived number of edges is predominantly two for $30 \mathrm{~ms}$ of duration while it is uniformly distributed across 2,3 and 4 for the largest duration.

\section{DISCUSSION}

Taken together, our results show that touch is very sensitive to the duration and sharpness (transition time) of short square ultrasonic pulses, which are in the range of a few milliseconds. Considering the existing evidence of tactile discrimination up to the molecular level [12], [17], such tactile acuity is not astonishing but raises the question of how the neural mechanisms mediating tactile perception integrate longer and more complex signals. The second performed experiment in this study shows that, depending on its duration, a square ultrasonic signal can be felt as one or two separate signals. Interestingly, the study also points out that the increase in the number of perceived edges is significantly less pronounced for sharper square signals while we expected that the sharper transitions would be more salient hence more noticeable by themselves. This might be due to the extremely fast transition time of $0.5 \mathrm{~ms}$ that can be achieved with the EViTa. It is possible that the transition is so transient that most tactile receptors, which have a typical bandwidth from 2 to 400 $\mathrm{Hz}$ [18], are incapable of encoding the rising and falling friction associated with the transitions of the ultrasonic lubrication.
Considering also that the typical unconstrained speed of exploration ranges from 5 to $20 \mathrm{~cm} / \mathrm{s}$ [19], it means that tactile features of 0.5 to $2 \mathrm{~cm}$ could be felt ambiguously during interaction with the tactile display. These findings also contribute to a current major challenge of tactile displays with haptic feedback, which is the definition a textel [13] or taxel [20] similarly to the definition of the pixel for visual feedback. The certainty to provide unambiguous tactile feedback is essential for the conception of sub-threshold frictional blocks, which are unnoticeable by themselves, but can be scaled and combined to induce a perceivable tactile pattern. Overall, this study is a first investigation on the influence of two parameters affecting the subjective tactile perception of short square reductions of friction: its sharpness and duration. For a better understanding of the subjective perception of friction-based haptic feedback in future studies, the behavioral (speed, normal force, lateral force,...) and technical parameters (waveform, intensity, periodicity,...) would need to be monitored in order to assess their influence on the human perception of transient frictional cues.

\section{ACKNOWLEDGMENT}

This work was founded by the FP7 Marie Curie Initial Training Network PROTOTOUCH, grant agreement No. 317100 and has been supported by IRCICA USR 3380 Univ.Lille - CNRS (www.ircica.univ-lille1.fr). The authors would like to thank Michel Amberg who prepared and calibrated the ultrasonic tactile display used in this study.

\section{REFERENCES}

[1] J. Monnoyer, E. Diaz, C. Bourdin, and M. Wiertlewski, "Ultrasonic Friction Modulation While Pressing Induces a Tactile Feedback," vol. 9775, no. July, Eds. Cham: Springer International Publishing, EuroHaptics 2016, pp. 171-179, 2016.

[2] S.-C. Kim, A. Israr, and I. Poupyrev, "Tactile rendering of 3D features on touch surfaces," Proc. 26th Annu. ACM Symp. User interface Softw. Technol. - UIST'13, pp. 531-538, 2013.

[3] A. Moungou, E. Vezzoli, C. Lombart, B. Lemaire-Semail, J.-L. Thonnard, and A. Mouraux, "A novel method using EEG to characterize the cortical processes involved in active and passive touch," IEEE Haptics Symposium (HAPTICS) 2016, pp. 205-210, 2016.

[4] D. J. Meyer, M. A. Peshkin, and J. E. Colgate, "Fingertip friction modulation due to electrostatic attraction," World Haptics Conf. WHC 2013, pp. 43-48, 2013.

[5] M. Biet, F. Giraud, and B. Lemaire-Semail, "Squeeze film effect for the design of an ultrasonic tactile plate," IEEE Trans. Ultrason. Ferroelectr. Freq. Control, vol. 54, no. 12, pp. 2678-2688, Dec. 2007.

[6] T. Sednaoui, E. Vezzoli, B. Dzidek, B. Lemaire-Semail, C. Chappaz, and M. Adams, "Experimental evaluation of friction reduction in ultrasonic devices," IEEE World Haptics Conf. WHC 2015, pp. 37-42, 2015.

[7] M. Wiertlewski, R. Fenton Friesen, and J. E. Colgate, "Partial squeeze film levitation modulates fingertip friction.," Proc. Natl. Acad. Sci. U. S. A., vol. 113, no. 33, pp. 9210-9215, 2016.

[8] E. Vezzoli, W. Ben Messaoud, M. Amberg, and M. Bueno, "Physical and Perceptual Independence of Ultrasonic Vibration and Electrovibration for Friction Modulation", IEEE transactions on haptics, vol. 8, no. 2, pp. 235-239, 2015.

[9] J. Platkiewicz, H. Lipson, and V. Hayward, "Haptic Edge Detection Through Shear.," Sci. Rep., vol. 6, no. November, p. 23551, 2015.

[10] M. Biet, G. Casiez, F. Giraud, and B. Lemaire-Semail, "Discrimination of virtual square gratings by dynamic touch on friction based tactile displays," Symp. Haptics Interfaces Virtual Environ. Teleoperator Syst. 2008 - Proceedings, Haptics, pp. 41-48, 2008 . 
[11] T. Sednaoui, E. Vezzoli, D. Gueorguiev, M. Amberg and B. LemaireSemail "Psychophysical Power Optimization of Friction Modulation for Tactile Interfaces", vol. 9775, Eds. Cham: Springer International Publishing, EuroHaptics 2016, pp. 354-362, 2016.

[12] D. Gueorguiev, S. Bochereau, A. Mouraux, V. Hayward, and J.-L. Thonnard, "Touch uses frictional cues to discriminate flat materials," Sci. Rep., vol. 6, no. April, p. 25553, 2016.

[13] D. J. Meyer, M. A. Peshkin, and J. E. Colgate, "Tactile Paintbrush : A Procedural Method for Generating Spatial Haptic Texture.", IEEE Haptics Symposium (HAPTICS) 2016, pp. 0-5, 2016.

[14] E. Vezzoli, T. Sednaoui, M. Amberg, F. Giraud, and B. LemaireSemail, "Texture Rendering Strategies with a High Fidelity Capacitive Visual-Haptic Friction Control Device," vol. 9775, no. July, pp. 251-260, 2016.

[15] M. Amberg, F. Giraud, B. Semail, P. Olivo, G. Casiez, and N. Roussel, "STIMTAC: A Tactile Input Device with Programmable Friction," in Proceedings of the 24th Annual ACM Symposium Adjunct on User Interface Software and Technology, pp. 7-8, 2011,

[16] F. A. A. Kingdom and N. Prins, "Psychophysics: A Practical Introduction", Elsevier academic press, 2009.

[17] L. Skedung, M. Arvidsson, J. Y. Chung, C. M. Stafford, B. Berglund, and M. W. Rutland, "Feeling small: exploring the tactile perception limits.," Sci. Rep., vol. 3, p. 2617, 2013.

[18] S. J. Bolanowski, "Four channels mediate the mechanical aspects of touch," J. Acoust. Soc. Am., vol. 84, no. 5, p. 1680, 1988

[19] T. Callier, H. P. Saal, E. C. Davis-Berg, and S. J. Bensmaia, "Kinematics of unconstrained tactile texture exploration.," $J$. Neurophysiol., p. jn.00703.2014, 2015.

[20] Y. Rekik, E. Vezzoli, L. Grisoni, and F. Giraud, "Localized Haptic Texture : A Rendering Technique Based on Taxels for High Density Tactile Feedback," CHI, vol. Proc. of C, 2017. 